\title{
Improving of Feldspar Efficiency As Potassium Fertilization For Potato (Salenum Tuberosumi) By Using Silicate Dissolving Bacteria And Compost
}

\author{
EL Shabrawy, R.M.I., *M.E, Aly., * O.H.M. El-Hoseiny, * and S.A Ismail,* \\ *Dept. of Soils and water, Fac. of Agric., Banha Univ. \\ **Soil, Water and Environment Res. Inst. A.R.C. \\ ***Agricultural engineering, Saudi, Arabia
}

\begin{abstract}
A field experiment was carried out at newly reclaimed land at El-Wady Co: Farm West of Wady El-Natron, El- Bohera Governorate, Egypt during the summer season of 2015 to evaluate the effect of different levels of mineral potassium sulphate and natural rock $\mathrm{K}$ and their combination under some bio-activators (without addition of bioactivators, with silicate dissolving bacteria and 10 ton compost/fed) on potato plant. The results reveal that increasing potassium level improved all of the studied growth parameter (shoots fresh and dry weights/plant), yield components (number of tubers/plant and mean tuber weight), yields (shoots and tuber fresh and dry yields ton/fed) and N, P and $\mathrm{K}$ uptake in tuber and shoots. Addition of feldspar enhanced all of the studied growth paramters, yield and its components and N P K uptake, Combination of $75 \%$ of the recmmonded rate of potassium sulphate with $25 \%$ feldspar gave all of the studied quality and quantity parameters equal to those obtained under $100 \%$ potassium sulphate. Bio-activators enhanced all of the abovementioned studied parameters. Compost was more effective than silicate dissolving bacteria in its effect on potato quality and quantity. The treatment of $75 \%$ of the recommended rate of potassium sulphate $+25 \%$ feldspar +10 ton/fed compostgave the highest values of potato quality and quantity parameters.
\end{abstract}

Key words : Potato, feldspar, potassium sulphate, silicate dissolving bacteria, compost, growth, yield and NPK uptake.

\section{Introduction}

Potato is one of the most important foods and cash crops cultivated worldwide under a wide range of climatic condition. At present it is the fourth most important food crop in terms of its production in the world, after wheat, rice and maize. Potato is considered among the highly recommended food security crop that can safeguard low-income countries from the risks posed by rising international food prices. In the recent years potato is becoming one of the key components in the livelihood systems in the world. Potatoes are often believed to have a high nutritional value that provides a major source of inexpensive energy from starch in many developing countries, and is one of six major crops providing $80 \%$ of human caloric intake worldwide (Leff, et al., 2004). Potato contains several vitamins, especially vitamin $\mathrm{C}$, and some important B group vitamins, it rich in minerals such as potassium and iron, a source of phenolic compounds that may have an important role in health, almost free of soluble sugar, a source of high quality protein, although deficient in the essential amino acid methionine, readily digested but also has a high water content so weight for weight there is a relatively low impact on blood sugar. In Egypt potato is one of the most important crops.
The potato was introduced to Egypt during the 1800 's, and the large scale cultivation the began during the First World War, when British colonial officials encouraged its production to feed their troops

Potassium is particularly important to obtain large productions. It has an important role in the synthesis of sugars and starch, which can be considered as the reason for the high requirement of this element. Because of its high mobility, potassium assists in the glucose transfer to the tubers. Potassium also diminishes frost damage, while it acts as a solute in the cell cytoplasm, thus low runn the freezing point of the cells. The element exercises a great influence on the plant's water balance as it effectively defends the assimilation tissues against drought damages and assures the uninterrupted sugars and starch development. Potassium also has a strong influence on the potato's texture, coloring and flavour as well as on its conservation as it provides more skin firmness. Lower doses of potassium resulted in more reductive sugar contents in the tubers and increase the gray coloring of the pulp. While, a high absorption of this element will diminish these symptoms, it also improves the resistance to hits and storage. The high prices of these fertilizers are responsible for increasing production cost and environmental pollution. 
The use of natural potassium fertilizer is low cost resources for providing plants with $\mathrm{K}$ which could alternate the expensive applied Kchemical fertilizers (Manning, 2010 and Labib et al., 2012. The main source of $\mathrm{K}$ for plants growing under natural conditions comes from the weathering of $\mathrm{K}$ minerals, e.g., $\mathrm{K}$-feldspar, K-mica and illite (Hellal et al., 2009). Many investigators concluded that K-feldspar may be valuable as a slow releasing fertilizer and cheaper source of K (Shafeek et al., 2005, Hellal et al., 2009; Manning, 2010, Labib et al., 2012 and Abou-el-Seoud, 2012).

The use of potassium solubilizing bacteria (KSB) as bio-fertilizer, i.e. silicate dissolving bacteria was suggested as a sustainable solution to improve plant growth, nutrition, root growth, plant competitiveness and responses to external stress factors (Vessey, 2003 and Priyanka and Sindhu, 2013). Moreover, KSB plays an important role in the formation of humus in soil, the cycling of other minerals tied up in organic matter (Zakaria, 2009). Also, it can able to solubilize rock - $\mathrm{K}$ mineral powder, such as mica, elite and orthoclases (feldspar) through the production and excretion of organic acids or chelate silicon ions to bring $\mathrm{K}$ into solution (Ullman et al., 1996 and Bennett et al., 1998). On the other hand, inoculation with potassium solubilizing bacteria, with either potassium sulfate or feldspar might provide faster and continuous supply of $\mathrm{K}$ for improved plant growth, yield and its quality (Eweda et al., 2007, and Abdel-Salam and Shams, 2012).

Organic manures, especially compost have been intensively used at the last decates to ameliorate soil fertility and consequently growth and production of cultivated crops. The decomposition of organic manure produces organic acids which plays an important role for solubilize the natural fertilizers and in turn increasing available $\mathrm{P}$ and $\mathrm{K}$ from the added rocks (Mohmoud and Hafez, 2004 and Bokhtor and Sakuri, 2005). Therefore, the objective of this work was to evaluate the possibility of substituting partly or totally the expensive potassium chemical fertilizers such as potassium sulfate by natural rock, i.e. feldspar with or without bio fertilizers such as silicate dissolving bacteria or compost , which solubilize potassium, and the effect of these treatments on the growth, yield and its component and nutrients uptake by potato plants.

\section{Materials and methods:}

A field experiment was conducted in newly reclaimed land at El wady Co. Farm, West of Wady El Natron, El Bohara Governorate, Egypte, during the summer season of 2015 to study the effect of application of natural rock potassium (K-feldspar) as a source of potassium for substituting the potassium sulphate fertilizer either partly or totaly under some bio-activators such as silicate dissolving bacteria i.e Bacillus circulans (SDB) and compost on growth characters, yield and yield component and nutrients status of potatoes (Solanum tuberosum), cv Diamond. A control treatment was also don i.e without addition of bioactivators.

The experimental design was split plot design involving two factors in four replication, Potassium treatments were set up in the main plots, while bio-activators were arranged in sub plot. Surface soil sample was taken from the experimental field to determine some physical propertis according to (Klute, 1982) and chemical propertis according to Page et al 1982) which are showen in Table1. Also, some chemical propertis of the compost used in the experiment were determined according Page et al (1982) and are showen in Table (2)

Table 1. Some physical and chemical properties of experimental soil before planting.

\begin{tabular}{lc}
\hline Soil properties & Value \\
\hline Particle size distribution & \\
Clay \% & 4.1 \\
Silt \% & 8.9 \\
Sand \% & 87 \\
Texture & Sand \\
Ca CO3 \% & 26.6 \\
Organic matter & 0.07 \\
pH (1:2.5 soil water-suspention & 8.16 \\
EC (dSm-1, soil paste extract) & 1.75 \\
Soluble cations (soil paste, meq /100g) & \\
$\mathrm{K}+$ & 0.26 \\
\hline $\mathrm{Na}+$ & 1.83 \\
$\mathrm{Ca}++$ & 8.93 \\
$\mathrm{Mg}++$ & 6.75 \\
\hline
\end{tabular}




\begin{tabular}{lc}
\hline Table 1 . Cont. & \\
\hline Soluble anions (soil paste, meq/100g) & \\
CO3- & 0.00 \\
HCO3- & 5.11 \\
CL- & 9.31 \\
SO4- $\quad 3.35$ & 3.35 \\
Available N (ppm) & 3.11 \\
Available P (ppm) & 7.25 \\
Available K (ppm) & 22.19 \\
\hline
\end{tabular}

Table 2. Some chemical properties of the compost used in the study:

\begin{tabular}{lc}
\hline Chemical propertis & Value \\
\hline $\mathrm{pH}(1: 2.5$ soil water suspention $)$ & 8.35 \\
$\mathrm{EC}$ dsm $^{-1}(1: 5)$ soil -water extraction & 3.95 \\
Organic matter $(\%)$ & 37.27 \\
Organic carbone $(\%)$ & 21.62 \\
Total nitrogen $(\%)$ & 1.46 \\
Total phosphorus $(\%)$ & 0.51 \\
Total potassium $(\%)$ & 1.29 \\
C/N ratio & $1: 14.8$ \\
\hline
\end{tabular}

\section{Field experiment:}

- Soil salt was leached for 10 days before planting using center pivot irrigation system. After partial drying, the soil was prepared with a chisel plow. The field was divided into $1.8 \mathrm{~m}$ wide strips. For each treatment two strips $(1.8 \mathrm{~m})$ were divided into $25 \mathrm{~m}$ long sections. Total plot area was $45 \mathrm{~m}^{2}(1.8 \mathrm{~m} \times$ $25 \mathrm{~m})$.

- Potato seed pieces (received from Holland) were cut (approximately $50 \mathrm{~g}$ pieces and cured in a dark storage at $15^{\circ} \mathrm{C}$ for 10 days. All seed pieces were treated with the moncut. Seed pieces were mechanically planted on 11 January 2015 using a two -row planter (structural potatoes planter) with $20 \mathrm{~cm}$ between hills and $90 \mathrm{~cm}$ between rows in all plots, Treatments:

1- Potassium was added at seven rates :

$\begin{array}{ll}\text { a- } & \text { Zero } \\ \text { b- } & 60 \mathrm{gk} \mathrm{K} / \text { fed as potassium sulfate. } \\ \text { c- } & 90 \mathrm{~kg} \mathrm{~K} / \text { fed as potassium sulfate. } \\ \text { d- } & 120 \mathrm{~kg} \mathrm{~K} / \text { fed as potassium sulfate. }\end{array}$

E-120 kg K / fed as feldspar

F- $60 \mathrm{~kg} \mathrm{~K} / \mathrm{fed}$ as potassium sulfate +60

$\mathrm{kg} /$ fed as feldspar.

G- $90 \mathrm{~kg} \mathrm{~K} /$ fed as potassium sulfate +30 $\mathrm{kg} /$ fed as feldspar.

2- Bio- activators was added as follow:

a- Without addition

b- Inoculation with silicate dissolving bacteria (SDB)

c- Compost (10 ton/fed)

Time of fertilizers application:

Compost was applied per plot during soil preparation.

- Potato pieces were inoculated with SDB immediately before sowing.

- K-feldspar (contain about $10.2 \%$ total K) was recived by Al-Ahram Mining and Natural Fertilizer Company in Egypt. Feldspar was added to the soil before planting, at soil preparation, while potassium sulphate was added at two doses i.e 50\% during soil preparation and the other $50 \%$ was added through the sprinkler irrigation regime through all growth season.

\section{Recorded data:}

\section{1- Plant growth:}

Five plants were taken at random from each plot after 75 days from planting and the fresh and dry weights/plant $(\mathrm{g})$ were determined

2-Yield and yield componant:

Five plants from each plot were randomly taken at harvest (after 120 days from planting) as a represented samples for determining the following characters:

- Total number of tubers per plant.

- Average of tuber weight $(\mathrm{g})$.

- Shoots and tubers fresh and dry yields for each plot expressed as ton/fed.

3- Nutrient contents:

Nitrogen, phosphorus and potassium concentration were determined in shoots and tubers according to the official methods described in A.O.A.C (1985).

4-Nutrient uptake was calcoulated as: nutrient concentration multiplied by root or foliage dry weight.

Statistical analyses:

The obtained data were statistically analyzed for variance according to Snedecor and Cochran (1980), significance of differences among treatments were compared using least 
significant differences (L.S.D) at 0,05 level probability.

\section{Results}

Growth parameters

Data in Table 3 represent the main effect of potassium the treatments on growth parameters of potato, i.e., shoot fresh and dry weights. Data reveal that shoot fresh and dry weights were gradually increased as potassium rate increased. Application of $120 \mathrm{~kg} \mathrm{~K} / \mathrm{fed}$ as potassium sulphate increased both fresh and dry weights by 55.8 and $45.6 \%$ compared with non potassium fertilization. The combined application of $30 \mathrm{~kg} \mathrm{~K} / \mathrm{fed}$ as feldspar with 90 $\mathrm{kg} \mathrm{K} / \mathrm{fed}$ as potassium sulphate gave shoot fresh and dry weights statistically equal to those obtained under $120 \mathrm{~kg} \mathrm{~K} / \mathrm{fed}$ as potassium sulphate. The lowest shoot fresh and dry weights were obtained under without potassium fertilization.

As for the main effect of bio-activators, results in Table 4 reveal that shoot fresh and dry weights of potato plants were significantly affected by the studied bio-activators. The effect of bio-activators on shoot fresh and dry weight could be arranged on the descending order as follow: 10 ton/fed compost > silicate dissolving bacteria > without bio-activators. Adahtion 10 ton compost/fed gave shoot fresh weight more than that obtained under SDB and without bio-activators by 13.4 and $19.7 \%$, respectively. Similar trend was obtained for shoot dry weight With regard to the interaction effect data in Table 5 show that SDB treatment was not affect shoot fresh and dry weights in absence of feldspar. However, inaculation potato seeds with SDB had a pronounced effect on shoot fresh and dry weights in presence of natural K-rock. The treatment of $120 \mathrm{~kg} \mathrm{~K} / \mathrm{fed}$ as potassium sulphate +10 ton compost/fed or $90 \mathrm{~kg} \mathrm{~K} / \mathrm{fed}$ as potassium sulphate $+30 \mathrm{~kg}$ $\mathrm{K} /$ fed as feldspar +10 ton compost/fed gave the heaviest shoot fresh and dry weights. On the other hand, the treatment of without both potassium and bio-activators gave the lowest shoot fresh and dry weights.

\section{Yield component parameters:}

Data of the effect of potassium sources and levels and bio-activators and their interaction on yield components of potato, namely, number of tubers/plant and the mean tuber weight are given in Tables 3, 4 and 5 .

As for the main affect of potassium treatments, results indicate that increasing potassium fertilization rate a markedly improved the studied potato yield components. Application of $120 \mathrm{~kg} \mathrm{~K} / \mathrm{fed}$ as potassium sulphate increased the studied potato yield components, where number of tubers/plant and tuber weight were increased by 28.3 and $14.0 \%$ compared with non-potassium application, respectively.

Table 3. The main effect of potassium sources and levels on shoots fresh and dry weights/plant at 75 days age as well as number of tuber /plant, tuber weight and fresh and dry yields of shoots and tuber ( $\mathrm{t} / \mathrm{fed})$ at harvest

\begin{tabular}{|c|c|c|c|c|c|c|c|c|}
\hline $\begin{array}{l}\text { Potassium } \\
\text { (kg K/fed) }\end{array}$ & $\begin{array}{l}\text { Shoot } \\
\text { fresh } \\
\text { weight/ } \\
\text { plant }(\mathrm{g})\end{array}$ & $\begin{array}{c}\text { shoot } \\
\text { dry } \\
\text { weight/ } \\
\text { plant }(\mathrm{g})\end{array}$ & $\begin{array}{c}\text { Number } \\
\text { of } \\
\text { tuber/plant }\end{array}$ & $\begin{array}{l}\text { Tuber } \\
\text { weight } \\
\text { (g) }\end{array}$ & $\begin{array}{l}\text { shoot } \\
\text { fresh } \\
\text { yield } \\
\text { (t/fed) }\end{array}$ & $\begin{array}{l}\text { shoot dry } \\
\text { yeield } \\
(t / f e d)\end{array}$ & $\begin{array}{l}\text { tuber } \\
\text { fresh } \\
\text { yield } \\
\text { (t/frd) }\end{array}$ & $\begin{array}{l}\text { tubers } \\
\text { dry yield } \\
(\mathrm{t} / \mathrm{fed})\end{array}$ \\
\hline Without & 447.30 & 44.10 & 10.80 & 50.86 & 7.95 & 0.93 & 7.10 & 1.09 \\
\hline $\begin{array}{l}60 \mathrm{~kg} \mathrm{~K} / \text { fed as } \\
\text { potassium sulphate }\end{array}$ & 457.70 & 45.60 & 11.94 & 53.96 & 11.41 & 1.42 & 11.54 & 1.42 \\
\hline $\begin{array}{l}90 \mathrm{~kg} \mathrm{~K} / \mathrm{fed} \text { as } \\
\text { potassium sulphate }\end{array}$ & 556.20 & 51.80 & 12.54 & 56.30 & 13.68 & 1.52 & 12.45 & 1.51 \\
\hline $\begin{array}{l}120 \mathrm{~kg} \mathrm{~K} / \text { fed as } \\
\text { potassium sulphate }\end{array}$ & 696.70 & 64.20 & 13.86 & 59.96 & 14.43 & 1.68 & 14.63 & 1.69 \\
\hline 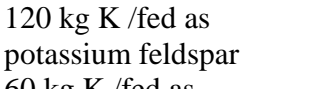 & 605.70 & 56.70 & 13.06 & 56.12 & 12.90 & 1.53 & 12.45 & 1.56 \\
\hline $\begin{array}{l}60 \mathrm{~kg} \mathrm{~K} / \mathrm{fed} \text { as } \\
\text { potassium sulphate }+60 \\
\mathrm{~kg} \mathrm{~K} / \text { fed as potassium } \\
\text { feldspar }\end{array}$ & 649.60 & 60.30 & 13.20 & 55.59 & 11.12 & 1.54 & 13.53 & 1.54 \\
\hline $\begin{array}{l}90 \mathrm{~kg} \mathrm{~K} / \text { fed as } \\
\text { potassium sulphate }+30 \\
\mathrm{~kg} \mathrm{~K} / \text { fed as potasium } \\
\text { feldspar }\end{array}$ & 685.75 & 63.10 & 13.72 & 58.22 & 14.95 & 1.67 & 14.60 & 1.63 \\
\hline L.S.D at 0.05 & 13.28 & 2.01 & 0.45 & 0.97 & 0.37 & 0.07 & 0.35 & 0.05 \\
\hline
\end{tabular}




\begin{tabular}{|c|c|c|c|c|c|c|c|c|}
\hline Bio-activators & 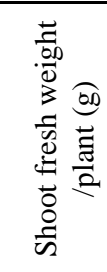 & 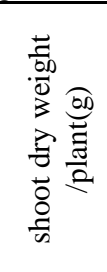 & 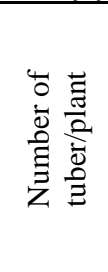 & 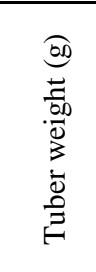 & 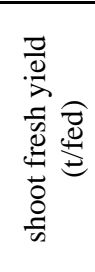 & 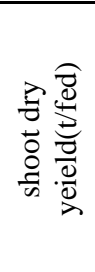 & 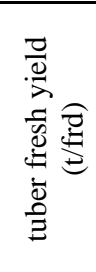 & 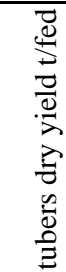 \\
\hline $\begin{array}{l}\text { Without } \\
\text { silicate } \\
\text { dissolving }\end{array}$ & 542.80 & 50.60 & 11.97 & 54.71 & 11.46 & 1.39 & 10.84 & 1.43 \\
\hline bacterya & 572.60 & 54.70 & 12.28 & 65.18 & 12.62 & 1.50 & 12.36 & 1.50 \\
\hline Compost & 649.50 & 60.30 & 13.95 & 56.69 & 14.15 & 1.64 & 14.49 & 1.62 \\
\hline L.S.D at 0.05 & 14.25 & 2.26 & 0.51 & 1.06 & 0.39 & 0.05 & 0.38 & 0.04 \\
\hline
\end{tabular}

Table 5. The effect of the interaction between potassium treatments and bio- activators on shoot fresh and dry weights/plant (g) at 75 days age as well as number of tubers/plant, tuber weight and fresh and dry yields of ahoots and tubers(t/fed) at harvest

\begin{tabular}{|c|c|c|c|c|c|c|c|c|c|}
\hline \multicolumn{2}{|c|}{ Treatments } & \multirow{2}{*}{ 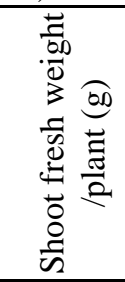 } & \multirow{2}{*}{ 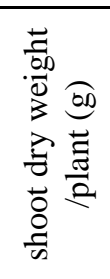 } & \multirow{2}{*}{ 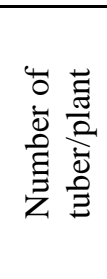 } & \multirow{2}{*}{ 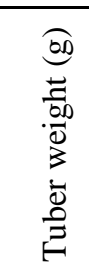 } & \multirow{2}{*}{ 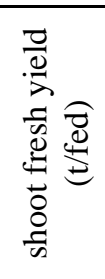 } & \multirow{2}{*}{ 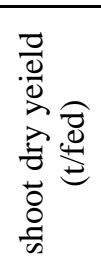 } & \multirow{2}{*}{ 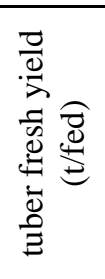 } & \multirow{2}{*}{ 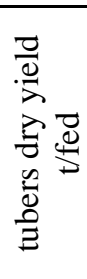 } \\
\hline $\begin{array}{l}\text { Potassium } \\
\text { ( kg K / fed) }\end{array}$ & Bio-activators & & & & & & & & \\
\hline \multirow{3}{*}{ Zero } & Without & 417.70 & 40.20 & 10.80 & 50.16 & 9.82 & 1.33 & 9.69 & 1.30 \\
\hline & SDB & 419.80 & 40.40 & 10.36 & 50.37 & 11.86 & 1.33 & 9.72 & 1.31 \\
\hline & Compost & 506.80 & 51.60 & 11.70 & 52.05 & 13.02 & 1.44 & 11.58 & 1.43 \\
\hline \multirow{3}{*}{$\begin{array}{l}60 \mathrm{~kg} \mathrm{~K} / \text { fed as } \\
\text { potassium sulfate }\end{array}$} & Without & 451.30 & 43.50 & 11.23 & 53.19 & 11.10 & 1.35 & 10.89 & 1.34 \\
\hline & SDB & 436.90 & 44.00 & 11.36 & 53.77 & 12.12 & 1.35 & 10.96 & 1.35 \\
\hline & Compost & 524.50 & 49.20 & 13.23 & 55.43 & 13.51 & 1.57 & 13.07 & 1.57 \\
\hline \multirow{3}{*}{$\begin{array}{l}90 \mathrm{~kg} \mathrm{~K} / \mathrm{fed} \text { as } \\
\text { potassium sulfate }\end{array}$} & Without & 514.50 & 48.30 & 11.88 & 55.97 & 12.70 & 1.43 & 11.78 & 1.44 \\
\hline & SDB & 516.80 & 48.50 & 11.90 & 56.91 & 12.72 & 1.44 & 11.85 & 1.46 \\
\hline & Compost & 638.70 & 58.70 & 13.85 & 57.03 & 14.63 & 1.70 & 13.71 & 1.63 \\
\hline \multirow{3}{*}{$\begin{array}{l}120 \mathrm{~kg} \mathrm{~K} / \text { fed as } \\
\text { potassium sulfate }\end{array}$} & Without & 676.30 & 62.20 & 12.70 & 57.31 & 14.02 & 1.65 & 13.23 & 1.65 \\
\hline & SDB & 678.60 & 62.40 & 12.81 & 57.46 & 14.05 & 1.65 & 17.73 & 1.67 \\
\hline & Compost & 734.70 & 67.60 & 16.03 & 59.11 & 15.22 & 1.74 & 17.28 & 1.75 \\
\hline \multirow{3}{*}{$\begin{array}{l}120 \mathrm{~kg} \mathrm{~K} / \text { fed as } \\
\text { feldspar }\end{array}$} & Without & 515.30 & 48.40 & 17.24 & 24.85 & 11.41 & 1.41 & 10.93 & 1.41 \\
\hline & SDB & 614.30 & 56.50 & 13.15 & 56.16 & 12.96 & 1.52 & 12.58 & 1.50 \\
\hline & Compost & 688.80 & 63.30 & 13.79 & 57.37 & 14.33 & 1.65 & 13.77 & 1.62 \\
\hline \multirow{6}{*}{$\begin{array}{l}60 \mathrm{~kg} \mathrm{~K} / \text { fed as } \\
\text { potassium sulfate }+ \\
60 \mathrm{~kg} \mathrm{~K} / \mathrm{fed} \text { as } \\
\text { feldspar } \\
90 \mathrm{~kg} \mathrm{~K} / \mathrm{fed} \text { as } \\
\text { potassium sulfate }+ \\
30 \mathrm{~kg} \mathrm{~K} / \mathrm{fed} \mathrm{as} \\
\text { feldspar }\end{array}$} & Without & 577.60 & 54.20 & 12.68 & 54.26 & 11.75 & 1.41 & 11.83 & 1.42 \\
\hline & SDB & 647.40 & 59.60 & 13.07 & 55.67 & 13.46 & 1.58 & 13.90 & 1.57 \\
\hline & Compost & 722.60 & 66.30 & 13.85 & 55.85 & 14.15 & 1.63 & 14.83 & 1.62 \\
\hline & SDB & 641.80 & 59.00 & 12.71 & 57.22 & 13.89 & 1.52 & 12.46 & 1.52 \\
\hline & Silicate & 677.10 & 62.30 & 13.78 & 58.45 & 14.15 & 1.65 & 14.13 & 1.64 \\
\hline & Compost & 73.63 & 67.70 & 15.18 & 59.01 & 15.20 & 1.74 & 17.20 & 1.73 \\
\hline L.S.D. at $0.05 \%$ & & 16.17 & 3.35 & 0.6 & 1.2 & 0.81 & 0.15 & 0.73 & 0.13 \\
\hline
\end{tabular}

The combined applicate of $90 \mathrm{~kg} \mathrm{~K} / \mathrm{fed}$ as potassium sulphate with $30 \mathrm{~kg} \mathrm{~K} / \mathrm{fed}$ as feldspar gave values of yield components equal to those obtained under $120 \mathrm{~kg} \quad \mathrm{~K} / \mathrm{fed}$ as potassium sulphate. Concerning the main effect of bioactivators, data clearly show that the studied bio-activators significantly increased potato yield components. Compost application at a rate of $10 \mathrm{ton} / \mathrm{fed}$ gave the highest number of tubers/plant and tuber weight, followed by silicate dissolving bacteria, whereas, nonbioactivators treatment gave the lowest values of number of tubers/plants and tuber weight,

As for the interaction between treatments, data show that number of tubers/plant and tuber weight responded to the interaction between potassium and bioactivators. The SDB affected potato yield components only in presence of feldspar. The highest values of number of tubers/plant and 
tuber weight achieved under the treatment of $120 \mathrm{~kg} \mathrm{~K} / \mathrm{fed}$ as potassium sulphate +10 ton/fed compost or $90 \mathrm{~kg} \mathrm{~K} / \mathrm{fed}$ as potassium sulphate $+30 \mathrm{~kg} \mathrm{~K} / \mathrm{fed}$ as feldspar +10 ton/fed compost. On the other hand, the lowest values of potato yield component parameters were recorded under the treatment of without potassium and no bio-activators application.

\section{Potato yields.}

The main effect of potassium sources and levels on potato yields in term of shoot fresh and dry yields, and tuber fresh and dry yields is shown in Table 3. Data show that all studied potato yield parameters were significantly increased due to increasing the chemical potassium sulphate levels up to $120 \mathrm{~kg} \mathrm{~K} / \mathrm{fed}$. Addition of $30 \mathrm{~kg} \mathrm{~K} / \mathrm{fed}$ as feldspar plus $90 \mathrm{~kg}$ $\mathrm{K} / \mathrm{fed}$ as potassium sulphate had the same effect as the high chemical fertilizer dose on potato yields. The non addition of potassium fertilizer gave the lowest potato fresh and dry shoots and tuber yield.

As the main effect of bio-activators, data in Table 4 indicate that using bio-activators increased shoots and tuber fresh and dry yields. The effect of bio-activators on potato yields could be arranged in the descending order as follow: 10 ton/fed composte $>$ silicate dissolving bacteria > without bio-activators. The relative increasing of shoot fresh yield, shoot dry yield, tuber fresh yield and tuber dry yield caused by $10 \mathrm{t} / \mathrm{fed}$ compost reached to 23.6, 18.0, 33.7 and $13.3 \%$ when compared with without bio-activators.
Considering the interaction affect, data in Table 5 indicate that potato yields significantly responded to the interaction between potassium sources and levels and bio-activators, where under chemical potassium fertilizer, SDB did not affect potato yields, whereas inoculation potato plants with silicate dissolving bacteria enhanced potato yield parameters only in the presence of natural K-rock. In general, the highest shoots and tubers fresh and dry yields were achieved under the treatment of $120 \mathrm{~kg}$ $\mathrm{K} / \mathrm{fed}$ as potassium sulphate +10 ton compost/fed or $90 \mathrm{~kg} \mathrm{~K} / \mathrm{fed}$ as potassium sulphate $+30 \mathrm{~kg} \mathrm{~K} / \mathrm{fed}$ as feldspar +10 ton compost/fed. On the other hand, the treatment of without both potassium and bio-activators application gave the lowest potato yield parameters $\mathrm{N}, \mathrm{P}$ and $\mathrm{K}$ uptake

Data presented in Table 6 show the main effect of potassium sources and levels on $\mathrm{N}, \mathrm{P}$, and $\mathrm{K}$ in shoots and /or tubers. The obtained results reveal that as chemical potassium fertilizer levels increased, the $\mathrm{N}, \mathrm{P}$ and $\mathrm{K}$ uptake in shoots and/or tubers were increased. The highest values of $\mathrm{N}, \mathrm{P}$ and $\mathrm{K}$ uptake were recorded under fertilized potato plants with 120 $\mathrm{kg} \mathrm{K} / \mathrm{fed}$ as potassium sulphate, followed by 90 $\mathrm{kg} \mathrm{K} / \mathrm{fed}$ as potassium sulphate $+30 \mathrm{~kg} \mathrm{~K} 2 / \mathrm{fed}$ as feldspar with no significant difference between them. On the other hand, the treatment of no potassium fertilization gave the lowest nutrient uptake.

Table 6. Interaction effect between potassium treatments and bio- activators on N,P and K uptake in shoot and /or tuber

\begin{tabular}{|c|c|c|c|c|c|c|c|c|c|}
\hline \multirow{2}{*}{$\begin{array}{l}\text { Potassium } \\
\text { (kg K/fed) }\end{array}$} & \multicolumn{3}{|c|}{ uptake in shoots } & \multicolumn{3}{|c|}{ uptake in tubers } & \multicolumn{3}{|c|}{ total uptake } \\
\hline & $\begin{array}{c}\mathrm{N} \\
\mathrm{kg} / \mathrm{fed}\end{array}$ & $\begin{array}{c}\mathrm{P} \\
\mathrm{kg} / \mathrm{fed}\end{array}$ & $\begin{array}{c}\mathrm{K} \\
\mathrm{kg} / \mathrm{fed}\end{array}$ & $\begin{array}{c}\mathrm{N} \\
\mathrm{kg} / \mathrm{fed}\end{array}$ & $\begin{array}{c}\mathrm{P} \\
\mathrm{kg} / \mathrm{fed}\end{array}$ & $\begin{array}{c}\mathrm{K} \\
\mathrm{kg} / \mathrm{fed}\end{array}$ & $\begin{array}{c}\mathrm{N} \\
\mathrm{kg} / \mathrm{fed}\end{array}$ & $\begin{array}{c}\mathrm{P} \\
\mathrm{kg} / \mathrm{fed}\end{array}$ & $\begin{array}{c}\mathrm{K} \\
\mathrm{kg} / \mathrm{fed}\end{array}$ \\
\hline Without & 35.0 & 4.7 & 23.7 & 24.6 & 7.7 & 28.2 & 92.6 & 12.4 & 56.8 \\
\hline $\begin{array}{l}60 \mathrm{~kg} \mathrm{~K} / \mathrm{fed} \text { as } \\
\text { potassium sulphate }\end{array}$ & 43.3 & 4.9 & 43.6 & 62.2 & 8.1 & 33.0 & 105.6 & 13.1 & 67.6 \\
\hline $\begin{array}{l}90 \mathrm{~kg} \mathrm{~K} / \mathrm{fed} \text { as } \\
\text { potassium sulphate }\end{array}$ & 52.1 & 5.5 & 40.6 & 69.4 & 8.6 & 35.9 & 122.2 & 13.8 & 76.4 \\
\hline $\begin{array}{l}120 \mathrm{~kg} \mathrm{~K} / \mathrm{fed} \text { as } \\
\text { potassium sulphate }\end{array}$ & 65.2 & 5.9 & 46.8 & 80.0 & 9.6 & 40.9 & 145.5 & 15.6 & 87.7 \\
\hline $\begin{array}{l}120 \mathrm{~kg} \mathrm{~K} / \mathrm{fed} \text { as } \\
\text { potassium feldspar }\end{array}$ & 53.4 & 5.2 & 37.3 & 67.0 & 8.8 & 35.8 & 120.2 & 14.3 & 73.0 \\
\hline $\begin{array}{l}60 \mathrm{~kg} \mathrm{~K} / \mathrm{fed} \text { as } \\
\text { potassium sulphate } \\
+60 \mathrm{~kg} \mathrm{~K} / \mathrm{fed} \text { as } \\
\text { feldspar }\end{array}$ & 49.0 & 5.3 & 40.5 & 70.8 & 9.0 & 36.8 & 119.8 & 114.3 & 72.8 \\
\hline $\begin{array}{l}90 \mathrm{~kg} \mathrm{~K} / \mathrm{fed} \text { as } \\
\text { potassium sulphate } \\
+30 \mathrm{~kg} \mathrm{~K} / \text { fed as } \\
\text { feldspar }\end{array}$ & 63.3 & 5.6 & 45.4 & 77.2 & 9.4 & 39.6 & 140.7 & 15.0 & 85.0 \\
\hline L.S.D at 0.05 & 4.5 & 0.5 & 2.9 & 4.2 & 0.3 & 2.05 & 6.1 & 0.6 & 4.1 \\
\hline
\end{tabular}


Concerning the nutrient uptake as affected by bio-activators, data in Table 7 show that the $\mathrm{N}, \mathrm{P}$ and $\mathrm{K}$ uptake by shoots and tubers as well as the total uptake were significantly responded to the studied bio activators. The effect of bioactivators on nutrient uptake could be arranged on desceding order as follow: 10 ton compost/fed > SDB > without bio-activators. Addition of 10 ton /fed compost gave total N,P and $\mathrm{K}$ uptake exceed that occurred by without bio-activators application by 24.7, 28.6 and $23.8 \%$, respavtivaly.

Concerning the effect of the interaction between potassium treatments and bioactivators on nutrient uptake, data in Table 8 indicate that inoculation potato plants with silicate dissoliving bactria did not affect $\mathrm{N}, \mathrm{P}$ and $\mathrm{K}$ uptake in absence of feldspar, while it enhanced nutrinent uptake in the case of application of $\mathrm{K}$ bearing rock. In general, the treatment of 120 $\mathrm{kg} \mathrm{K}$ as potassium sulphate +10 ton compost/fed or $90 \mathrm{~kg} \mathrm{~K}$ as potassium sulphate + $30 \mathrm{~kg} \mathrm{~K}$ as potassium feldspar +10 ton/fed compost gave the highest values of $\mathrm{N}, \mathrm{P}$ and $\mathrm{K}$ uptake by shoots and/or tuber, whereas, the lowest nutrients uptake were obtained under the without potassium fertilization + without bioactivators.

Table 7. The main effect of bio-activators on N, P and K uptake in shoots and/or tuber

\begin{tabular}{|c|c|c|c|c|c|c|c|c|c|}
\hline \multirow{2}{*}{$\begin{array}{c}\text { Bio- } \\
\text { activators }\end{array}$} & \multicolumn{3}{|c|}{ uptake in shoot } & \multicolumn{3}{|c|}{ uptake in tubers } & \multicolumn{3}{|c|}{ total uptake } \\
\hline & $\begin{array}{c}\mathrm{N} \\
(\mathrm{kg} / \mathrm{fed})\end{array}$ & $\begin{array}{c}\mathrm{P} \\
(\mathrm{kg} / \mathrm{fed})\end{array}$ & $\begin{array}{c}\mathrm{K} \\
(\mathrm{kg} / \mathrm{fed})\end{array}$ & $\begin{array}{c}\mathrm{N} \\
(\mathrm{kg} / \mathrm{fed})\end{array}$ & $\begin{array}{c}\mathrm{P} \\
(\mathrm{kg} / \mathrm{fed})\end{array}$ & $\begin{array}{c}\mathrm{K} \\
(\mathrm{kg} / \mathrm{fed})\end{array}$ & $\begin{array}{c}\mathrm{N} \\
(\mathrm{kg} / \mathrm{fed})\end{array}$ & $\begin{array}{c}\mathrm{P} \\
(\mathrm{kg} / \mathrm{fed})\end{array}$ & $\begin{array}{c}\mathrm{K} \\
(\mathrm{kg} / \mathrm{fed})\end{array}$ \\
\hline Without & 45.60 & 4.60 & 34.90 & 63.70 & 8.00 & 33.30 & 109.50 & 12.60 & 68.20 \\
\hline SDB & 44.40 & 4.90 & 37.20 & 67.20 & 8.50 & 34.70 & 116.60 & 13.30 & 71.80 \\
\hline Compost & 60.10 & 6.30 & 44.50 & 76.60 & 9.80 & 39.20 & 136.60 & 16.20 & 84.40 \\
\hline $\begin{array}{l}\text { L.s.D at } \\
0.05\end{array}$ & 2.90 & 0.20 & 2.60 & 3.10 & 0.30 & 2.50 & 5.40 & 0.60 & 3.50 \\
\hline
\end{tabular}

Table 8. Interaction effect between potassium treatments and bio- activators on N,P and $\mathrm{K}$ uptake in shoots and /or tubers

\begin{tabular}{|c|c|c|c|c|c|c|c|c|c|c|}
\hline \multirow[b]{2}{*}{$\begin{array}{l}\text { Potassum } \\
\text { treatments }\end{array}$} & \multirow{2}{*}{ 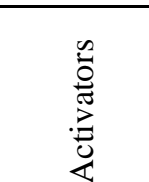 } & \multicolumn{3}{|c|}{ uptake in shoots } & \multicolumn{3}{|c|}{ uptake in tubers } & \multicolumn{3}{|c|}{ total uptake } \\
\hline & & $\begin{array}{c}\mathrm{N} \\
\mathrm{kg} / \mathrm{fed}\end{array}$ & $\begin{array}{c}\mathrm{P} \\
\mathrm{kg} / \mathrm{fed}\end{array}$ & $\underset{\mathrm{kg} / \mathrm{fed}}{\mathrm{K}}$ & $\begin{array}{c}\mathrm{N} \\
\mathrm{kg} / \mathrm{fed}\end{array}$ & $\begin{array}{c}\mathrm{P} \\
\mathrm{kg} / \mathrm{fed}\end{array}$ & $\underset{\mathrm{kg} / \mathrm{fed}}{\mathrm{K}}$ & $\begin{array}{c}\mathrm{N} \\
\mathrm{kg} / \mathrm{fed}\end{array}$ & $\begin{array}{c}\mathrm{P} \\
\mathrm{kg} / \mathrm{fed}\end{array}$ & $\begin{array}{c}\mathrm{K} \\
\mathrm{kg} / \mathrm{fed}\end{array}$ \\
\hline \multirow{3}{*}{ SDB } & Without & 30.90 & 4.10 & 25.60 & 53.80 & 7.20 & 26.80 & 84.70 & 11.40 & 52.50 \\
\hline & Silicate & 30.70 & 4.50 & 25.90 & 54.10 & 7.30 & 26.80 & 84.90 & 11.60 & 52.60 \\
\hline & Compost & 43.80 & 5.50 & 34.60 & 64.50 & 8.60 & 30.90 & 108.10 & 14.20 & 65.40 \\
\hline $60 \mathrm{~kg} \mathrm{~K} / \mathrm{fed}$ as & Without & 38.50 & 4.30 & 31.40 & 56.80 & 7.50 & 30.80 & 95.60 & 11.90 & 62.20 \\
\hline potassium & SDB & 38.60 & 4.30 & 31.50 & 57.20 & 7.50 & 30.90 & 95.80 & 11.90 & 62.10 \\
\hline sulfate & Compost & 52.90 & 6.00 & 40.90 & 72.60 & 9.40 & 37.30 & 125.40 & 15.50 & 78.10 \\
\hline $90 \mathrm{~kg} \mathrm{~K} / \mathrm{fed}$ as & Without & 48.00 & 4.70 & 37.50 & 64.90 & 7.90 & 33.60 & 113.20 & 12.70 & 71.20 \\
\hline potassium & SDB & 48.50 & 4.80 & 37.70 & 65.50 & 8.20 & 33.90 & 113.80 & 12.90 & 71.40 \\
\hline sulfate & Compost & 61.50 & 6.00 & 46.50 & 77.90 & 9.80 & 40.10 & 139.60 & 19.90 & 86.60 \\
\hline $120 \mathrm{~kg} \mathrm{~K} / \mathrm{fed}$ as & Without & 61.90 & 5.40 & 43.50 & 77.50 & 9.20 & 39.30 & 139.50 & 14.70 & 82.90 \\
\hline potassium & SDB & 62.30 & 5.50 & 43.70 & 77.80 & 9.30 & 39.50 & 139.80 & 14.80 & 83.00 \\
\hline sulfate & Compost & 71.50 & 6.90 & 52.90 & 84.60 & 10.40 & 43.70 & 155.80 & 17.30 & 97.20 \\
\hline $120 \mathrm{~kg} \mathrm{~K} / \mathrm{fed}$ as & Without & 43.90 & 4.40 & 32.80 & 60.50 & 7.90 & 33.00 & 104.20 & 12.30 & 65.90 \\
\hline potassium & SDB & 54.20 & 4.80 & 37.10 & 56.90 & 8.60 & 35.20 & 120.00 & 13.50 & 72.20 \\
\hline feldspar & Compost & 62.00 & 6.30 & 42.00 & 74.70 & 10.00 & 39.10 & 136.50 & 16.30 & 80.90 \\
\hline $60 \mathrm{~kg} \mathrm{~K} / \mathrm{fed}$ as & Without & 40.40 & 4.50 & 34.60 & 62.00 & 7.90 & 33.70 & 102.40 & 17.50 & $68 . .2$ \\
\hline $\begin{array}{l}\text { potassium } \\
\text { sulfate }+60 \mathrm{~kg}\end{array}$ & SDB & 49.30 & 4.90 & 40.70 & 72.40 & 9.00 & 37.30 & 121.70 & 13.90 & 77.90 \\
\hline $\begin{array}{l}\mathrm{K} / \text { fed as } \\
\text { feldspar }\end{array}$ & Compost & 57.20 & 6.40 & 46.30 & 78.10 & 10.10 & 39.50 & 135.40 & 16.60 & 85.70 \\
\hline $90 \mathrm{~kg} \mathrm{~K} /$ fed as & Without & 56.30 & 4.70 & 39.20 & 70.30 & 8.20 & 35.70 & 126.00 & 17.90 & 74.80 \\
\hline $\begin{array}{l}\text { potassium } \\
\text { sulfate }+30 \mathrm{~kg}\end{array}$ & SDB & 62.20 & 5.30 & 43.80 & 77.60 & 9.30 & 39.50 & 140.10 & 14.70 & 83.30 \\
\hline $\begin{array}{l}\mathrm{K} / \text { fed as } \\
\text { feldspar }\end{array}$ & Compost & 71.40 & 6.80 & 53.10 & 83.80 & 10.60 & 43.50 & 155.60 & 17.30 & 96.75 \\
\hline L.S.D. at $0.05 \%$ & & 4.6 & 0.7 & 3.2 & 5.7 & 0.6 & 2.3 & 8.3 & 0.9 & 6.2 \\
\hline
\end{tabular}




\section{Discussion}

On the basis of the experimental result, it coull be stated that all of the studied growth parameters of potato (shoot fresh and dry weights/plant at 75 days age) were significantly affected by increasing the rate of the applied potassium fertilizer. This may be attributed to that potassium is necessary for mineral lignin and cellulose development which gives strength and stiffness to plants, enabling them to stand upright with reduced lodging. Potassium encourages plant root development, as an example, plants well supplied with potassium have abundance of roots to utilize soil moisture efficiency, whereas plants grown under low potassium levels have very few root, thereby resulting in low water use efficiency. Increaseing root growth improves the drought resistance of most plants (Mengle and Kirk by (1987). they added that potassium is an essential macronutrient and most abundantly absorbed cation that plays an important role in the growth, metabolism and development of plants. Without adequate potassium, the plants will have poorly developed roots, grow slowly, produce small seeds and have lower yields. Gardener et al (1985) reported that potassium was found to serve a vital role in photosymthesis by direct increase in growth and leaf area index and hence $\mathrm{CO} 2$ assimilation and increasing the outward translocation of photospnthates. These results are in accordance with those reported by Hashem et al (2016) and Zelelew et al (2016)

Yields and yield attributes at harvest (number of tuber/plant, mean of tuber weight, shoot and tuber fresh and dry yield ton/fed were significantly responded to potassium fertilization. Increasing potassium rate gradually increased yield and yield attributes of potato. The positive effect of potassium fertilization on yield and its attributes is mainly explained by its effect on growth parameters as the abovementioned discussion. In this concern, many investigators observed an increase in potato yields as a result of increasing the $\mathrm{K}$ fertilization level (Hashem et al, 2016), which attributed these increment in the yield of potato tubers either to formation of large sized tubers or increasing number of tubers per plant or both. Mengel and Kirk by (1987) indicated that $\mathrm{K}$ regulates the amount of water in the plant and plays a role in maintaining turgidity of plant cells and osmotic adjustment. In the absence of sufficient $\mathrm{K}$, crops less able to withstand water stress during drought season. Therefore, increesing potassium levels improved water relations of potato plants under water stress (Islam, 2004). Similar results were obtnied by
Abd El-Latif et al (2011) and Shutian et al (2015)

It is evident from the presented data that nitrogen, phosphorus and potassium uptake in shoots and tuber in potato plant after harvest were increased as mineral potassium levels increased. This increase could be explained on the basis of increasing the availibilty of nutrients in the soil. In addition, the increase of the nutrient content in shoots in response to the high rate of the added potassium may be due to the high mobility of $\mathrm{K}$ nutrient in plant. In this concern, Mengel and Kirk by (1987) mentioned that, as $\mathrm{NH}_{4}^{+}$is very similar to $\mathrm{K}^{+}$in the ionic radius, it can exchange with fixed $\mathrm{K}$ and vice versa, where each nutrient can enhance the availability of the other. Moreover, the enhancement of $\mathrm{N}, \mathrm{P}$ and $\mathrm{K}$ uptake by potato shoots and tubers due to $\mathrm{K}$ application is mainly due to its effect on shoot and tuber yield as discussed above. The obtained results are in agreement with those obtaing by Abd El-Latif et al (2011) who reported that N, P and K content increased as $\mathrm{K}$ levels increased.

Potassium bearing minerals are mainly as K-feldspar mica and illite (Labib et al, 2012). Many experiments have been carried in various countries emphasizing the importans of applying the K-bearing rocks to results substitute chemical fertilizers. Results of this investigation reveal that addition of feldspar solely significantly increased growth, yield and yield components parameters of potato as well as $\mathrm{N}, \mathrm{P}$ and $\mathrm{K}$ uptake when compared with no potassium fertilization solely, but its effect is lower than that of the highest level of chemical potassium fertilizer. The effectiveness of $\mathrm{K}$ bearing minerals as K-fertilizer is low, which mainly due to their slow availability. Combination of $30 \mathrm{~kg} \mathrm{~K} / \mathrm{fed}$ as feldspar with 90 $\mathrm{kg} \mathrm{K} / \mathrm{fed}$ as potassium sulphate gave similar effect as the higher dose of K-sulphate. These results are in line with those obtained by (Labib et al 2012).

Among the materials used in agriculture, fertilizer is the most widely used based on the production process, it can roughly categorized into three types: chemical, organic and biofertilizer. Each type of fertilizer has its advantages and disadvantages. These advantages need to be integrated in order to achieve optimum performances by each type of fertilizer and to realize balanced nutrient management for crop production. Many farmers and scientists in the world are becoming increasingly aware of the importance of organic production. Organic fertilizers, such as compost provide slow release of nutrients in soil (El-Etr et al, 2004 and Mohmoud and Hafez, 2010). The organic fertilizer has many advantages 
such as, the nutrient supply is more balanced, enhance soil biological activity; enhance root growth due to better soil structure, increase the organic matter content of soil, therefore improving the exchange capacity nutrients, promoting soil aggregates and buffering the soil against acidity; alkalinity; salinity; pesticides and toxic heavy metals, release nutrients slowly, contribute to the residual of organic $\mathrm{N}$ and $\mathrm{P}$ in the soil, reducing $\mathrm{N}$ leaching loss and $\mathrm{P}$ fixation, encourage the growth of beneficial microorganisms and earthwerms, and help to suppress certain plant diseases and soil born diseases and parasites (Bokhtair and Sakuri 2005). It is clearly evident from the obtained data that compost improved all of the studied growth, yield and yield component and $\mathrm{N}, \mathrm{P}$ and $\mathrm{K}$ uptake in potato plants, which mainly due to improving soil properties, soil organic matter and soil fertility (Abou-Hussein, 2005). The obtained results are similar to those obtained by (Abo-Zeid and bakry 2010) and ElSayed et al (2014).

Modern crop production depends upon industrial fertilizers that are more expensive, and also cause pollution and farmland degradation. The use of plant promoting rhizobacteria (PGPR), including potassium solubilizing bacteria (KSB) as bio-fertilizers, has been suggested as a sustainable solution to improve plant nutrient and production (Vessey, 2003). KSB is a heterotrophic bacterium, which obtaine all their energy and celular carbon from pre-existing organic materials. Thus, it is important in formation of humus in soil, the rceycling of other minerals tied in organic matter, and the prevention of buildup of dead organic material (Zakaria, 2009). Moreover, KSB are able to solubilize rock $\mathrm{K}$ mineral powder, such as micas, illite and orthoclases (feldspar), It was shown that KSB, such as bacillas mueilaginosus increased $\mathrm{K}$ availability in soil and increased mineral content in plant (Sheng and Huang 2002). However, B.mucilaginosus is also thought to accelerate the dissolution of a variety of silicate by the protection of extracellular polysaccharides (EPS). Liu (2006) proved that polysaccharides strongly adsorbed the organic acids and attached to the surface mineral, resulted in an area of high concentration of organic acids near the mineral. He reported that the EPS adsorb $\mathrm{SiO}_{2}$ and this affects the equilibrium between the mineral and fluid phases and led to the reaction toward $\mathrm{SiO}_{2}$ and $\mathrm{K}^{+}$solubilization. The results of this work show that, the main effect of silicate dissolving bacteria (SDB) on growth parameters, yield and yield components and $\mathrm{N}$, $\mathrm{P}$ and $\mathrm{K}$ uptake was significant, where they significantly increased when compared with non bio-activators application. The increase caused by SBD may be due to its ability to produce extensive root length (Shong and Huang, 2002) and improveing root development and enhance the rate of water and mineral uptake (Saghir et al 2007). Also, Ibrahim et al (2010) mentioned that the promotive affect of bio fertilizer may be due to the ability of $B$. mucilaginous to produce some growth promoting substances such as IAA, gibberellins and abscisic acid. It is also well known that these bacteria produce organic, inorganic acids and $\mathrm{CO}_{2}$ which lead to decrease soil $\mathrm{pH}$, hence converted insoluble forms of $\mathrm{P}$ and $\mathrm{K}$ into soluble ones ( Alexander,1997 and Wani et al 2007). The obtained results are in harmony with those obtained by EL-Sayed and Hassan (2014) and Shams and Fekry (2014).

Concerning the interaction effect between potassium levels and sources, and biostimulates, the obtained data indicated that, although potato productivity were significantly affected by inoculation with SDB, these biofertilizer did not affect potato productivity under chemical potassium sulphate treatments, but their positive effect occur only in the presence of feldspar. The increment in potato productivity owaing to inoculation with silicate dissolving bacteria under feldspar application may be due to that bacteria can solubilize the natural potassium sources provide faster and continuous supply of $\mathrm{K}$ for optimal plant growth (Abou-el-Seoud, 2012 and Priyanka and Simdha, 2013). The obtained results are in harmony with those obtaimed by Shams and Fekry (2014).

Also, it could be stated from the interaction effect between studied fertilizers that the treatment of $90 \mathrm{~kg} \mathrm{~K} / \mathrm{fed}$ as potassium sulphate $+30 \mathrm{~kg} \mathrm{~K} / \mathrm{fed}$ as feldspar +10 ton compost/fed is the more effective treatment for increasing potato productivity. The effect this treatment was similar to that obtained under the recommended chemical potassium fertilization. This may be due to the role of compost on reducing soil $\mathrm{pH}$ which in turn enhances the feldspar solubility (Abd El-Motagally (2009). This means that it could be save about $25 \%$ from the recommended dose of $\mathrm{K}$ through the the addition of feldspar and compost. On the other hand, potato plants without potassium and without bio-stimulators gave the lowest productivity. The obtained results are line with those obtained by Hellal et al (2009) and Hellal et al (2013)

\section{Conclusion}

From the results of this study, it is worthy to mention that there were positive 
effects of application of K-feldspar in combination with compost or silicate dissolving bacteria, where they increased the effect of feldspar on potato production by enhancing the solubility of rock feldspar and release $\mathrm{K}$ in available form. Thus it could be recommended to add $75 \% \mathrm{~K}$ as potassium sulphate $+25 \% \mathrm{~K}$ as feldspar +10 ton compost /fed or using SDB inoculant to produc maximum productivity of potato.

\section{References}

Abd El-Latif, K.M., E.A.M. Osmana, R. Abdullahb, and N. Abd- el Kader, 2011. Response of potato plants to potassium fertilizer rates and soil moisture deficit Advances in Applied Science Research, 2 (2): 388-397.

Abdel-Motagally, M.F. and K. Attia, 2009. Response of sugar beet plants to nitrogen and potassium fertilization in sandy calcareous soil. J Agric \& Bio., 11: 695-700

Abdel-Salam., M. A. and A. S. Shams, 2012. Feldspar-K fertilization of potato (Solanum tuberosum L.) augmented by bio-fertilizer. American-Eurasian J. Agric. \& Environ. Sci., 12 (6): 694-699.

Abou-el-Seoud., A. A, 2012. Impact of rock materials and biofertilization onm $\mathrm{P}$ and $\mathrm{K}$ availability for maize (Zea maize) under calcareous soil condition. Saudi Journal of Biological Science, 19: 55-63.

Abou-Hussein, S. D., I. I. El-Oksh., T. ElShorbagy., U. A. El-Behairy, 2005. Effect of chicken manure, compost and biofertilizers on vegetative growth, tuber characteristics and yield of potato crop. Egypt. J. Hortic., 29(1):135-149

Abou-Zeid, M.Y. and M.A.A. Bakry, 2011. Integrated effect of bio-organic manures and mineral fertilizers on potato productivity and the fertility status of a calcareous soil. Austr. J. Basic \& Applied Sci., 5(8): 1385-1399

Alexander, M., 1997. Introduction to Soil Microbiology. Second ed. John Wiley \& Sons Inc., New York.

AOAC, 1985. Official Methods of Analysis. 14th Edition, Association of Official Analytical Chemists, Washington DC, 43.292. 7.001, 7.009, 7.006

Bennett, P.C., W.J.Choi, and J.R. Rogera, 1998. Microbial destruction of feldspars. Mineral Management, 8: 149-50.

Bokhtiar, S.M, and K. Sakurai, 2005. Effect of application of inorganic and organic fertilizers on growth, yield and quality of sugarcane. sugar Tech, 7: 35-37.

El-Etr, S. H., A. Mueller., L.S. Tompkins., S. Falkow, and D.S. Merrell,
2004. Phosphorylation-independent effects of CagA during interaction between Helicobacter pylori and T84 polarized monolayers. J. Infect. Dis. 190, 1516-1523.

El-Sayed, F.S., A.H. Hassan, M. M. El-Mogy, 2014. Impact of bio- and organic fertilizers on potato Yield, quality and tuber weight loss after harvest European Association for Potato Research, (81): 58-67.

Eweda, W.E., S.M. Selim, M.I. Mostafa, and D.A. Abd El-Fattah, 2007. Use of Bacillus circulans as bio-accelerator enriching composted agricultural wastes identification and utilization of the microorganism for compost production. In : Proceedings of the 12th Conference of the Microbiology. Organized by the Egyptian Soc. Of App. Micro. (ESAM), Giza, Egypt, 18-20 pp. 4365 .

Gardner, F.P., R.B Pearce, and R. L. Mitchell, 1985. Physiology of crop plants. iowa university press, ames.

Hashem, F. A., N.M. Taha, and S.H. AbdElrahman, 2016. Effect of phosphate and potassium fertilizer rates on potato plants grown under water stress conditions, International Journal of Plant \& Soil Science 13(1): 2320-7035.

Hellal, F. A., M. Abd El-Hady, and A. A. M. Ragab, 2009. Influence of organic amendments on nutrient availability and uptake by faba bean plants fertilized by rock phosphate and feldspar. American- Euasian J. Agric.Environ. Sci. 6 (3): 271-27.

Hellal, F.A., S.A.A. El-Sayed, and R.M. Zewainy, 2013. Effects of natural potassium source enriched with compost on nutrient uptake and yield of sugar beet grown in newly reclaimed soils. Middle East Journal of Agriculture Research, (3): 101-107.

Ibrahim, H.I.M., M. M.A. Zaglol and A. M. M. Hammad 2010 Response of balady guava trees cultivated in sandy calcareous soil to biofertilization with phosphate dissolving bacteria and / or VAM Fung. J. Am. Sci., 6 (9): 399-404

Islam, M.S., M.M. Haque., M.M. Khan, T. Hidaka, and M.A. Karim. 2004. Effect of fertilizer potassium on growth, yield and water relations of bush bean (Phaseolus vulgaris L.) under water stress conditions. Japanese Journal of Agriculture, 48 (1):1-9.

Klute, A., 1982. Methods of Analysis part-1 physical and Mineralogical Methods (anded) American society of Agronomy, Medison. Win Wiscomsin, USA.

Labib, B. F., K. Ghabour., I. S. Rahim, and M. M. Wahba, 2012. Effect of potassium bearing rock on the growth and quality of 
potato crop (Solanum tuberosum). J. Agric. Biotech. Sustainable Dev, 4 (1), 7 -15.

Leff, B., N, Ramankutty, and J.A. Foley, 2004. Geographical distribution of major crops across the world. Global Biogeochemical Cycles, 18(1): B1009

Liu M, 2006. Cchl mediates calcium entry in Cryptococcus neoformans ans is essential in low-calcium environments. Eukaryot Cell 5(10): 1788-96.

Mahmoud, A. R, and M.M. Hafez, 2010. Increasing productivity of potato plants by using potassium fertilizer and humic acid application. International Journal of Academic Research, 2, 83-88.

Manning, D.A.C, 2010. Mineral sources of potassium for plant nutrition. A Review Agron. Sustain Dev. pp: 281-294.

Mengel, K, and E. A. Kirk, 1987. Principles of Plant Nutrition. International Potash Institute, Worblaufen-Bern, Switzerland.

Page, A.L., R.H. Miller, and D. R.Keeny, 1982. Methods of Soil Analysis, Part 2, Chemical and Microbiological Properties.S.S.S.A Publisher, Madison,

Priyanka, P, and S.S. Sindhu, 2013. Potassium solubilization by rhizosphere bacteria: influence of nutritional and environmental Conditions. Journal of Microbiology Research, 23(1): 25-3

Saghir, K.M., A. Zaidi, and P.A.Wani, 2007. Role of phosphate solubilizing microorganisms in sustainable agriculture: a review. Agron. Sustain. Dev. 27:29-43.

Shafeek, M.R., O.A.H. El-Zeiny, and M.E. Ahmed, 2005. Effect of natural phosphate and potassium fertilizer on growth, yield and seed composition of pea plant in new reclaimed soil. Asian J. plant Sci., (6): 608612.
Shams, A.S, and W.A. Fekry, 2014. Efficiency of applied K-feldspar with potassium sulphate and silicate dissolving bacteria on sweet potato

Zagazig j. Agric. Res., 41 (3): 467-477.

Sheng, X.F, and W.Y. Huang, 2002. Mechanism of potassium release from feldspare affected by the strain NBT of silicate bacterium. Acta Pedol. Sin., 39: 863-871.

Shutian, L. A., Y.U. Duanb, G. Tianwen., Z. Pingliang., H. Ping, J. Adrian, and S. Alexey, 2015. Potassium management in potato production in Northwest region of China. Field Crops Research, (174): 48-54.

Snedecor, G.W, and W.G. Cochran. 1980. Statistical methods. 7th ed. Lowa State Unv. Press., Ames., Lowa, U.S.A.

Ullman, W.J., D.L. Kirchman, and S.A. Welch, 1996. Laboratory evidence for micobially mediated silicate mineral dissolution in nature. Chem. Geol., 132: 11-17

Vessey, K.J, 2003. Plant growth promoting rhizobacteria as biofertilizers. Plant Soil, 255: 571-586.

Wani, P.A., M.S. Khan and A. Zaidi. 2007. Synergistic effects of the inoculation with nitrogen fixing and phosphate-solubilizing rhizobacteria on the performance of field grown chickpea. J. Plant Nutr. Soil Sci. 170: 283-287.

Zakaria, A.A.B, 2009. Growth optimization of potassium solubilizing bacteria isolated from biofertilizer. Bachelor of Cehm. Eng. (Biotech.) Fac. Cehm. Natural Resources Engl. Univ., Malaysia, Pahang, P40.

Zelelew, D.Z., S. Lall., T.T Kidane, and B.M. Ghebreslassie, 2016. Effect of potassium levels on growth and productivity of potato varieties ame- rican Journal of Plant Sciences. 7, 1629-1638. 


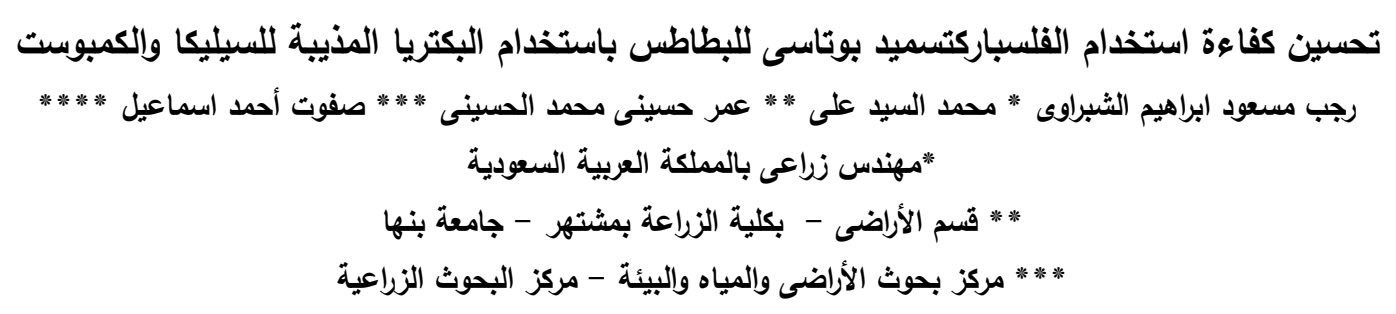

أجريت تجربة حقلية بالأراضى الجديدة بغرب وادى النطرون بمحافظة البحيرة ، مصر خلال موسم النمو الصيفى 2015 لتقييم تأثير أضافة

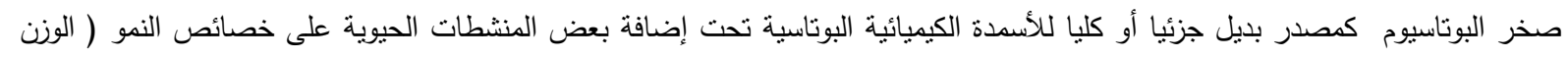

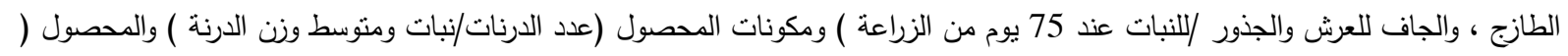

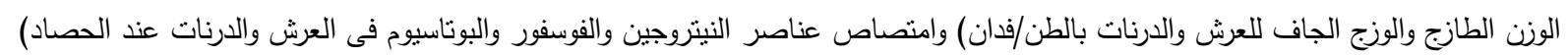

لمحصول البطاطس صنف دايموت

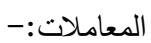

1- تمت أضافة البوتاسيوم بسبع معدلات :-

أ- صفر

ب - 60 كيلو جرام كبريتات بوتاسيوم / فدان

ت - 90 كيلو جرام كبرينات بوتاسيوم / فدان

ث - 120 كيلو جرام كبريتات بوتاسيوم / فدان

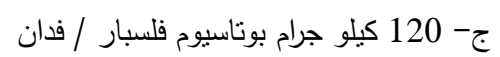

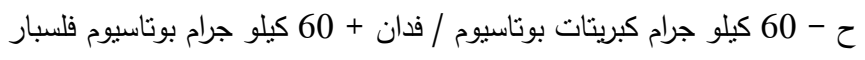

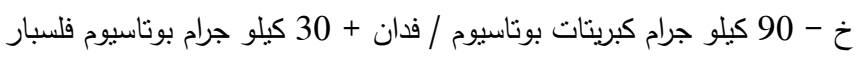

2- المنشطات :-

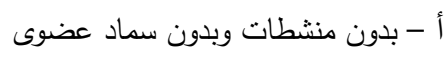

ب - التلقيح بيكتريا مذيبة للسيليكا

ج - عضوى ( كمبوست 10 طن/فدان )

وكانت أهم النتائج المتحصل عليون كان كما يلى :

صفات النمو

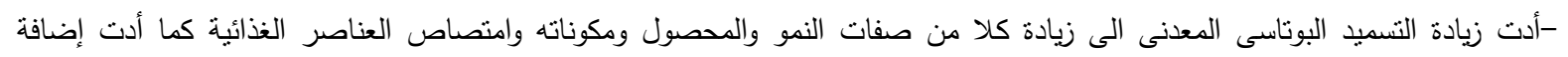

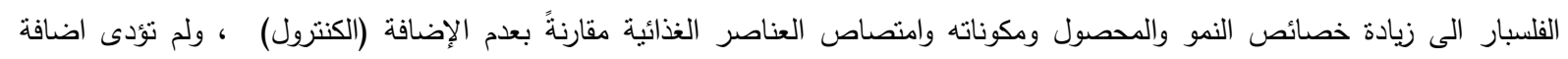

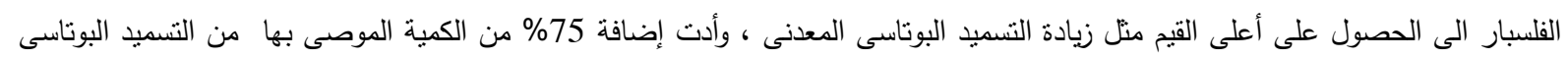

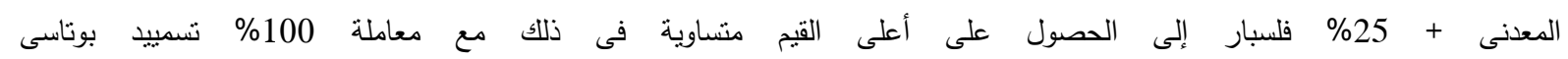

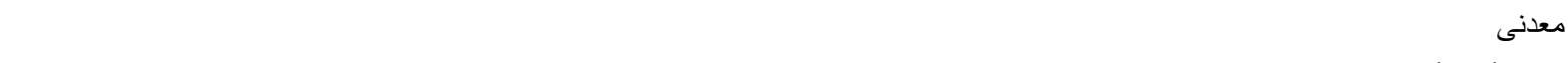

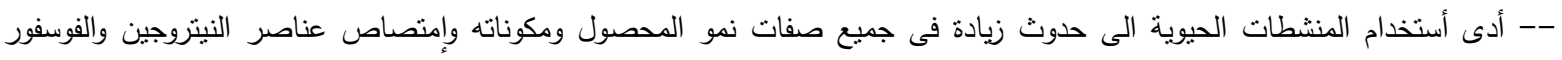

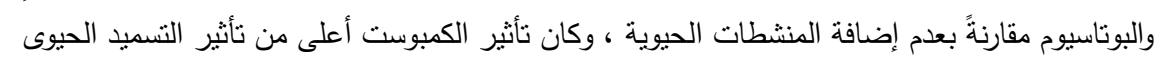

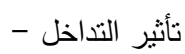

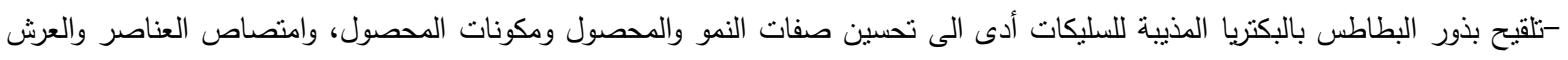

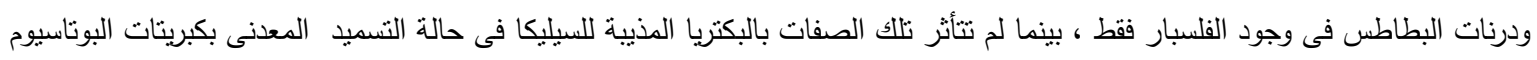

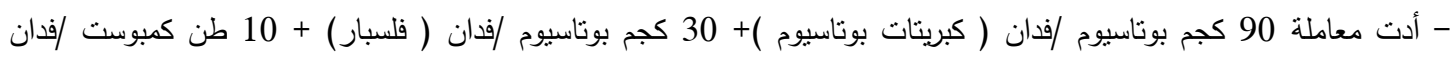

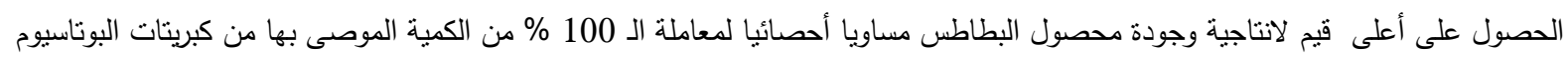

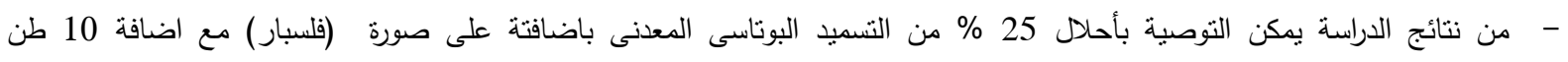

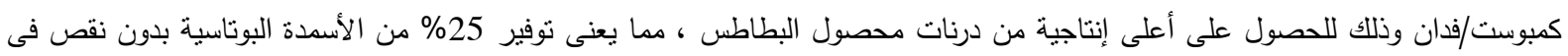
الإنتاجية . 